\title{
A COMPARISON PRINCIPLE FOR TERMINAL VALUE PROBLEMS IN ORDINARY DIFFERENTIAL EQUATIONS
}

\author{
BY
}

THOMAS G. HALLAM(1)

\begin{abstract}
A comparison principle for a terminal value problem of an ordinary differential equation is formulated. Basic related topics such as the existence of maximal and minimal solutions of terminal value problems are investigated. The close relationship between the existence of the extremal solutions of a terminal value problem and the concept of asymptotic equilibrium of the differential equation is explored. Several applications of the terminal comparison principle are given.
\end{abstract}

1. Introduction. The comparison principle has proved to be very useful in the study of various qualitative problems in ordinary differential equations. Previously, comparison principles have been formulated in terms of initial value problems and, in this setting, the applications are numerous. For an excellent discussion of the comparison principle and many of its applications, see the monograph by V. Lakshmikantham and S. Leela [6].

In this article the fundamental theory of a scalar comparison principle for a terminal value problem is developed. First, the concept of extremal solutions, which plays a basic role in any comparison principle, is discussed in a terminal setting. Asymptotic equilibrium of a differential equation is shown to be closely connected to the existence of these extremal solutions of terminal value problems.

A terminal comparison principle has been stated in [7]; however, the proof given there for the case of weak inequalities needs some modification. Since we require additional hypotheses as well as the hypotheses of Mamedov [7] in our principle below, the validity of the comparison principle in the full generality of [7] remains an open question.

2. Preliminary hypotheses and definitions. The solution of the initial value problem that consists of the scalar differential equation

$$
d r / d t=g(t, r)
$$

and the point $\left(t_{0}, r_{0}\right)$ will be denoted by $r\left(t, t_{0}, r_{0}\right)$. In (1), we assume that $g$ is

Received by the editors September 21, 1971.

AMS 1970 subject classifications. Primary 34A40, 34C10.

Key words and phrases. Comparison principle, differential inequality, maximal and minimal solutions, terminal value problem, asymptotic equilibrium.

(1)This research was supported in part by the National Science Foundation under grant GP-11543.

Copyright $\odot 1972$, American Mathematical Society 
continuous from $R_{+} \times R$ to $R$ where $R_{+}$denotes the nonnegative real numbers. It will be tacitly assumed that, given any $r_{0} \in R$, there exists a $t_{0} \in R_{+}$such that each solution $r\left(t, t_{0}, r_{0}\right)$ of $(1)$ exists on $\left[t_{0}, \infty\right)$ and $\lim _{t \rightarrow \infty} r\left(t, t_{0}, r_{0}\right)$ exists. $A$ solution of the terminal value problem that consists of equation (1) and a terminal value $r_{\infty}$ will be denoted by $r\left(t ; r_{\infty}\right)$. This terminal value problem will be written as $\left\{(1) ; r_{\infty}\right\}$.

A solution $r_{M}\left(t ; r_{\infty}\right)$ is the maximal solution of the terminal value problem $\left\{(1) ; r_{\infty}\right\}$ on the interval $I$ provided any other solution $u\left(t ; r_{\infty}\right)$ of the terminal value problem $\left\{(1) ; r_{\infty}\right\}$ which is valid on $I$ satisfies the inequality $u\left(t ; r_{\infty}\right) \leq$ $r_{M}\left(t ; r_{\infty}\right)$ for all $t \in I$. A solution $r_{m}\left(t ; r_{\infty}\right)$ of the terminal value problem $\left\{(1) ; r_{\infty}\right\}$ that exists on the interval $I$ is called the minimal solution of the terminal value problem $\left\{(1) ; r_{\infty}\right\}$ on $I$ provided any other solution $u\left(t ; r_{\infty}\right)$ of $\left\{(1) ; r_{\infty}\right\}$ satisfies $u\left(t ; r_{\infty}\right) \geq r_{m}\left(t ; r_{\infty}\right)$ for all $t$ in $I$. Either of these special types of solutions will be referred to as an extremal solution of the terminal value problem.

For initial value problems, the hypothesis $g \in C\left[R_{+} \times R, R\right]$ is sufficient to ensure the existence of maximal and minimal solutions $[6, p .11]$. However, even if the continuity of $g$ in $t$ is extended to the interval $[0, \infty]$, then extremal solutions of terminal value problems need not exist. For example, all of the solutions of the differential equation

$$
d r / d t=(t+1)^{-1}
$$

are convergent but the terminal value problem $\{(2) ; 0\}$ has no extremal solutions.

A topic that is closely related to the existence of extremal solutions of terminal value problems is asymptotic equilibrium. A. Wintner, in a sequence of papers [8], [9], [10], and F. Brauer [1], [2] have studied the asymptotic equilibrium problem; also, see [6, p. 88$]$.

The concept of an extremal solution is a local property of a differential equation. The close connection between extremal solutions and asymptotic equilibrium leads to the next definition which is a local analogue of asymptotic equilibrium. Equation (1) is locally in asymptotic equilibrium about $r_{\infty}$ provided there exists a constant $\delta>0$ with the property that whenever $r_{0}$ satisfies the inequality $\left|r_{0}-r_{\infty}\right|$ $<\delta$ then the terminal value problem $\left\{(1) ; r_{0}\right\}$ has a solution. It is clear that $(1)$ is in asymptotic equilibrium if it is locally in asymptotic equilibrium about $r_{\infty}$ for every $r_{\infty} \in R$.

3. Results on extremal solutions and asymptotic equilibrium.

Theorem 1. Suppose that initial value problems of (1) bave unique solutions. If (1) is locally in asymptotic equilibrium about $r_{\infty}$ then the terminal value problem $\left\{(1) ; r_{\infty}\right\}$ has both extremal solutions. 
Proof. If the terminal value problem $\left\{(1) ; r_{\infty}\right\}$ does not have a maximal solution, then there exists a sequence $\left\{t_{n}\right\}$, where $t_{n} \in R_{+}, n=1,2, \ldots$; and $\lim _{n \rightarrow \infty} t_{n}=\infty$, and there exists a constant $r_{1}>r_{\infty}$ such that the solution $r\left(t, t_{n}, r_{1}\right)$ of (1) satisfies $\lim _{t \rightarrow \infty} r\left(t, t_{n}, r_{1}\right)=r_{\infty}$. The hypothesis of the theorem implies that there exists a $\delta>0$ such that whenever $r_{0}$ satisfies the inequality $\delta>r_{0}>r_{\infty}$ then the terminal value problem $\left\{(1) ; r_{0}\right\}$ has a solution.

Choose any such $r_{0}$ with $r_{\infty}<r_{0}<r_{1}$. The integer $N$ may be chosen sufficiently large so that the solution $r\left(t ; r_{0}\right)$ is equal to the solution $r\left(t, t_{N}, r_{1}\right)$ for some $t \in R^{+}$. This contradicts the assumption that initial value problems for (1) are unique and shows that $\left\{(1) ; r_{\infty}\right\}$ has a maximal solution.

It can be shown in a similar manner that the terminal value problem $\left\{(1) ; r_{\infty}\right\}$ has a minimal solution. This completes the proof of Theorem 1 .

As an immediate consequence of the definition of asymptotic equilibrium and Theorem 1 we obtain the following:

Corollary. Suppose that initial value problems of (1) bave unique solutions. If (1) is in asymptotic equilibrium, then all terminal value problems have both extremal solutions.

Remark. The terminal value problem which consists of the differential equation

$$
d r / d t= \begin{cases}0, & r \geq 1, \\ -r+1, & r<1,\end{cases}
$$

Ind the terminal value $r_{\infty}=1$ has a maximal solution. However, (3) is not locally in asymptotic equilibrium about $r_{\infty}=1$. The example also demonstrates that a terminal value problem may have one extremal solution but not the other extremal solution.

In fact, it is possible for a terminal value problem to have both extremal solutions and yet not be locally in asymptotic equilibrium on either side of the terminal value. For example, the problem $\left\{r^{\prime}=-r(r-1)(r+1)\right.$; 0$\}$ has extremal solutions but is not locally in asymptotic equilibrium about $r_{\infty}=0$.

Theorem 2. If all terminal value problems of (1) bave both extremal solutions, then (1) is in asymptotic equilibrium.

Proof. If the conclusion of the theorem is false, then there is a terminal value problem $\left\{(1) ; r_{\infty}\right\}$ which has no solution. We assert that there must exist a constant $r_{M}$ where $r_{M}>r_{\infty}$ and with the property that the terminal value problem $\left\{(1) ; r_{M}\right\}$ has a solution. If this is not the case; then, since all solutions of (1) that are valid for large $t$ are convergent, there must be some terminal value problem of (1) that does not have a maximal solution. In a similar manner, it can be shown that there exists an $r_{m}, r_{m}<r_{\infty}$, such that $\left\{(1) ;{ }_{m}\right\}$ has a solution. 


\section{Define}

$r^{*}=\inf \left\{r_{0} \mid r_{M} \geq r_{0}>r_{\infty}\right.$ and the problem $\left\{(1) ; r_{0}\right\}$ has a solution $\}$. If $r^{*}=r_{\infty}$ then a sequence of solutions $\left\{r\left(t ; r_{n}\right)\right\}, n=1,2, \ldots$, can be constructed with the following properties:

(i) $\lim _{n \rightarrow \infty} r_{n}=r_{\infty}$;

(ii) $r\left(t ; r_{n}\right)$ is the minimal solution of the problem $\left\{(1) ; r_{n}\right\}$; and

(iii) $\left\{r\left(t ; r_{n}\right)\right\}$ converges uniformly to a function $r(t)$ on $[T, \infty)$.

Property (iii) follows from Ascoli's theorem by observing that the closure of the sequence $\left\{r\left(t ; r_{n}\right)\right\}$ is compact in the space of continuous functions on $[T, \infty)$ having limit at infinity, with the topology defined by the supremum norm.

For each $n=1,2, \ldots, r\left(t ; r_{n}\right)$ is a solution of the integral equation

$$
r\left(t ; r_{n}\right)=r_{n}-\int_{t}^{\infty} g\left(s, r\left(s ; r_{n}\right)\right) d s, \quad t \in R_{+} .
$$

Property (iii) implies that the limit of the sequence $r(t)$ is a solution of

$$
r(t)=r_{\infty}-\int_{t}^{\infty} g(s, r(s)) d s, \quad t \in R_{+} .
$$

Therefore, $r(t)$ is a solution of the terminal value problem $\left\{(1) ; r_{\infty}\right\}$. This contradicts our original assumption and implies that $r^{*}>r_{\infty}$.

If

$$
r_{*}=\sup \left\{r_{0} \mid r_{\infty}>r_{0} \geq r_{m} \text { and the problem }\left\{(1) ; r_{0}\right\} \text { has a solution }\right\},
$$

then an argument similar to the one above can be used to establish that $r_{*}<r_{\infty}$. Using the above techniques, it can also be shown that the terminal value problems $\left\{(1) ; r^{*}\right\}$ and $\left\{(1) ; r_{*}\right\}$ have solutions. Let $r^{*}(t)$ denote the minimal solution of $\left\{(1) ; r^{*}\right\}$ and $r_{*}(t)$ denote the maximal solution of $\left\{(1) ; r_{*}\right\}$.

Numbers $r_{0} \in R$ and $T \in R_{+}$can be chosen in such a manner that the solution $r\left(t, T, r_{0}\right)$ of (1) satisfies $r_{*}(t)<r\left(t, T, r_{0}\right)<r^{*}(t), t \geq T$. These inequalities lead to a contradiction of the definitions of $r^{*}$ or $r_{*}$. This means that the terminal value problem $\left\{(1) ; r_{\infty}\right\}$ has a solution; therefore, (1) is in asymptotic equilibrium.

4. A terminal comparison principle. Mamedov [7, Theorem 1] has stated a comparison principle similar to the result given below. As was mentioned previously, the second part of the proof in [7] requires some modification. The difficulties arise because the existence of a solution of the terminal value problem $\left\{(1) ; u_{0}\right\}$ need not imply that the terminal value problem $\{d u / d t=g(t, u)-1 / n$; $\left.u_{0}+1 / n\right\}, n=1,2, \cdots$, has a solution. For example, the differential equation (1) with $g \equiv 0$ illustrates this point.

In many instances, the asymptotic equilibrium of (1) is invariant when (1) is subjected to an integrable perturbation. Two situations in which this occurs are 
the following. We assume that (1) is in asymptotic equilibrium.

(i) If $g$ is nonnegative and nondecreasing in $r$ for each fixed $t \in R_{+}$, then the equation

$$
d u / d t=g(t, u)+b(t)
$$

is in asymptotic equilibrium for each $b \in L^{1}\left(R_{+}\right) \cap C\left[R_{+}, R\right]$. (This result follows from the Lemma in [4].).

(ii) If (1) is uniformly convergent in variation, then (4) is in asymptotic equilibrium for each $b \in L^{1}\left(R_{+}\right) \cap C\left[R_{+}, R\right]$. (This result is contained in [3].)

For later use in connection with the comparison principle, it is convenient to introduce the following terminology. The terminal value problems $\left\{(1) ; r_{\infty}\right\}$ allows perturbations in $L^{1}\left(R_{+}\right) \cap C\left[R_{+}, R\right]$ provided the equation (4) is locally in asymptotic equilibrium about $r_{\infty}$ for each $b \in L^{1}\left(R_{+}\right) \cap C\left[R_{+}, R\right]$.

In the next results, $D^{*}$ denotes the upper right Dini derivate.

Theorem 3 (Terminal Comparison Principle). Suppose that initial value problems of (1) have unique solutions. Suppose that the terminal value problem $\left\{(1) ; r_{\infty}\right\}$ allows perturbations in $L^{1}\left(R_{+}\right) \cap C\left[R_{+}, R\right]$. Let $r_{M}\left(t ; r_{\infty}\right)$ be the maximal solution of the terminal value problem $\left\{(1) ; r_{\infty}\right\}$ and suppose that $r_{M}\left(t ; r_{\infty}\right)$ exists on $J_{0}=\left[t_{0}, \infty\right)$. If $V(t)$ is a continuous function that satisfies the differential inequality

$$
D^{*} V(t) \geq g(t, V(t)), \quad t \in J_{0},
$$

and the terminal inequality

$$
V(\infty) \leq r_{\infty}
$$

then

$$
V(t) \leq r_{M}\left(t ; r_{\infty}\right), \quad t \in J_{0} .
$$

Proof. If all of the inequalities in both the hypothesis and the conclusion of Theorem 3 are replaced by strict inequalities, then the theorem is valid even if ${ }^{r} M\left(t ; r_{\infty}\right)$ is replaced by the minimal solution of the terminal value problem $\left\{(1) ; r_{\infty}\right\}$. See [7, Theorem 1] for the proof of this statement.

The terminal value problem

$$
\left\{d u / d t=g(t, u)-e^{\left.-t / n ; r_{\infty}+1 / n\right\}}\right.
$$

has a solution $u\left(t ; r_{\infty}+1 / n\right)$ provided $n$ is sufficiently large, say $n \geq N$.

From (5) and (5), it follows that

$$
D^{*} V(t)>g(t, V(t))-e^{-t / n,} V(\infty)<r_{\infty}+1 / n .
$$


The above result of Mamedov implies that

$$
V(t)<u\left(t ; r_{\infty}+1 / n\right), \quad t \in J_{0}, n \geq N .
$$

Proceeding as we did in the proof of Theorem 2, the sequence $\left\{u\left(t ; r_{\infty}+1 / n\right)\right\}$, $n \geq N$, can be shown to be uniformly convergent on $J_{0}$. Since the function $u\left(t ; r_{\infty}+1 / n\right)$ is a solution of the integral equation

$$
u\left(t ; r_{\infty}+\frac{1}{n}\right)=r_{\infty}+\frac{1}{n}-\int_{t}^{\infty} g\left(s, u\left(s ; r_{\infty}+\frac{1}{n}\right)\right) d s+\frac{e^{-t}}{n}, \quad t \in J_{0}
$$

it follows that $\lim _{n \rightarrow \infty} u\left(t ; r_{\infty}+1 / n\right)$ is a solution of the terminal value problem $\left\{(1) ; r_{\infty}\right\}$. The conclusion ( 7 ) now follows from the inequality (9) by letting $n$ go to infinity.

5. Applications. Let $\|\cdot\|$ denote some norm of $n$-dimensional Euclidean space, $R^{n}$. Suppose that for $x \in R^{n}$ and $t \in R_{+}$,

$$
\|f(t, x)\| \leq g(t,\|x\|)
$$

where $f \in C\left[R_{+} \times R^{n}, R^{n}\right], g \in C\left[R_{+} \times R_{+}, R_{+}\right]$, and $g(t, r)$ is nondecreasing in $r$ for each fixed $t \in R_{+}$. If it is assumed that

$$
\int^{\infty} t^{\sigma+1} g(t, \lambda) d t<\infty
$$

for all $\lambda>0$ and some $\sigma>0$ then all of the solutions of

$$
d x / d t=f(t, x)
$$

that are valid for large $t$ are convergent (see [2] and [4]). Suppose that a solution $x=x(t)$ of (12) has limit $x_{\infty}$, then the expression $e_{x}(t) \equiv\left\|x(t)-x_{\infty}\right\|$ will be called the error function associated with $x$. In many computational problems, it is useful to have an estimate on the magnitude of the error function. As a first application of the terminal comparison principle, we present a new result in this direction.

Theorem 4. Suppose that the functions $f$ and $g$ satisfy conditions (10) and (11). For each constant $c$, suppose that initial value problems of $d r / d t=$ $-g(t, r+c)$ bave unique solutions. Then, each solution $x=x(t)$ of (12) that is valid for large $t$ satisfies $t^{\sigma} e_{x}(t) \in L_{0}^{\infty}\left(R_{+}\right) \cap L^{p}\left(R_{+}\right)$for all $p \geq 1$.

Proof. From the definition of $e_{x}(t)$, it follows that $\|x(t)\| \leq e_{x}(t)+\left\|x_{\infty}\right\|$. Using this estimate and inequality (10) we obtain the terminal differential inequality

$$
\left\{D^{*} e_{x}(t) \geq-g\left(t, e_{x}(t)+\left\|x_{\infty}\right\|\right) ; e_{x}(\infty)=0\right\}
$$


Next, we note that the associated terminal value problem

$$
\left\{d r / d t=-g\left(t, r+\left\|x_{\infty}\right\|\right) ; 0\right\}
$$

allows perturbations in $L^{1}\left(R_{+}\right) \cap C\left[R_{+}, R\right]$. In fact, it can be shown that (11) implies that the equation $d r / d t=-g\left(t, r+\left\|x_{\infty}\right\|\right)$ is in asymptotic equilibrium. The proof is a standard application of the Schauder-Tychonoff fixed point theorem. It is similar to the proof of the Lemma in [4] and for this reason will be omitted.

It follows from Theorem 1 that the problem (13) has both extremal solutions. If $r_{M}(t ; 0)$ denotes the maximal solution of the problem (13) and $r_{M}(t ; 0)$ exists on $J=\left[t_{0}, \infty\right)$ then the Terminal Comparison Principle gives the inequality

$$
e_{x}(t) \leq r_{M}(t ; 0), \quad t \in J_{0}
$$

The solution $r_{M}(t ; 0)$ of the terminal value problem (13) must be a solution of the integral equation

$$
r_{M}(t ; 0)=\int_{t}^{\infty} g\left(s . r_{M}(s ; 0)+\left\|x_{\infty}\right\|\right) d s
$$

Since $g$ is nondecreasing and $r_{M}(t ; 0)$ is bounded, there exists some constant $B>0$ such that

$$
t^{\sigma} r_{M}(t ; 0) \leq t^{\sigma} \int_{t}^{\infty} g(s, B) d s .
$$

The right side of inequality (15) is in $L_{0}^{\infty}\left(R_{+}\right)$by virtue of (11). An integration of the right side of (15) shows that $t^{\sigma} r_{M}(t ; 0)$ is in $L^{1}\left(R_{+}\right)$. Therefore, $t^{\sigma} r_{M}(t ; 0)$ is in $L_{0}^{\infty}\left(R_{+}\right) \cap L^{p}\left(R_{+}\right)$for all $p \geq 1$. The combination of this result with inequality (14) gives the conclusion of the theorem.

Remark. Theorem 4 is an extension of a result in [5, Theorem 1]. A similar proof of Theorem 4 could also be given here. The idea to use the Terminal Comparison Principle in the proof of Theorem 4 originated in a discussion with Professor V. Lakshmikantham. The author also wishes to express his thanks to Professor Lakshmikantham for calling his attention to reference [7].

A weight function other than $t^{\sigma}$ can be used in (11) and a result analogous to Theorem 4 may be obtained.

For example, let $\phi=\phi(t)$ be a continuously differentiable function with $\phi^{\prime} \geq \delta>0$ on $R_{+}$and such that $\int^{\infty} \phi^{\prime}(t) g(t, \lambda) d t<\infty$ for all $\lambda>0$. Then, the maximal solution $r_{M}(t ; 0)$ of (13) satisfies $\phi(t) r_{M}(t ; 0) \in L_{0}\left(R_{+}\right) \cap L^{p}\left(R_{+}\right)$for all $p \geq 1$.

As another application of the Terminal Comparison Principle, a terminal analogue of the Perron Uniqueness Theorem [6, p. 48] is formulated.

Theorem 5. Suppose that $f \in C\left[R_{+} \times R^{n}, R^{n}\right], g \in C\left[R_{+} \times R_{+}, R_{+}\right]$, and 


$$
\left\|f\left(t, x_{1}\right)-f\left(t, x_{2}\right)\right\| \leq g\left(t,\left\|x_{1}-x_{2}\right\|\right)
$$

for all $t \in R_{+}, x_{1}, x_{2} \in R^{n}$. Suppose that the equation

$$
d r / d t=-g(t, r)
$$

bas unique solutions to initial value problems. Let the problem $\{(17) ; 0\}$ allow perturbations in $L^{1}\left(R_{+}\right) \cap C\left[R_{+}, R\right]$ and suppose that the only solution of $\{(17) ; 0\}$ is $r(t) \equiv 0$. Then, the terminal value problem $\left\{(12) ; x_{\infty}\right\}$ bas at most one solution.

Proof. Suppose that there exist two solutions $x_{1}(t), x_{2}(t)$ of the problem $\left\{(12) ; x_{\infty}\right\}$. Defining $m(t)=\left\|x_{1}(t)-x_{2}(t)\right\|$ and using (16), we obtain $D^{*} m(t) \geq$ $-g(t, m(t))$. Theorem 3 implies that $m(t) \leq 0$ which proves the theorem.

As a final application of the Terminal Comparison Principle, we present a terminal integral inequality whose initial analogue has been very useful in many qualitative problems in ordinary differential equations.

Theorem 6. Suppose that $g \in C\left[R_{+} \times R_{,} R_{+}\right]$and that $g(t, r)$ is nondecreasing in $r$ for each fixed $t \in R_{+}$. Let (17) bave unique solutions to initial value problems and suppose that the terminal value problem $\left\{(17) ; r_{\infty}\right\}$ allows perturbations in $L^{1}\left(R_{+}\right) \cap C\left[R_{+}, R\right]$. Let the maximal solution $r_{M}\left(t ; r_{\infty}\right)$ be defined on $J_{0}=$ $\left[t_{0}, \infty\right)$. If $m(t)$ is a continuous function defined on $J_{0}$, such that $m(t) \leq r_{\infty}+$ $\int_{t}^{\infty} g(s, m(s)) d s, t \in J_{0}$, then $m(t) \leq r_{M}(t), t \in J_{0}$.

Proof. By defining $V(t) \equiv r_{\infty}+\int_{t}^{\infty} g(s, m(s)) d s$, the hypothesis states that $m(t) \leq V(t)$ for $t \in J_{0}$. Also, $V^{\prime}(t) \geq-g(t, V(t))$ and $V(\infty)=r$. The Terminal Comparison Principle yields the conclusion.

\section{REFERENCES}

1. F. Braver, Global behavior of solutions of ordinary differential equations, J. Math. Anal. Appl. 2 (1961), 145-158. MR 24 \#A284.

2. - Bounds for solutions of ordinary differential equations, Proc. Amer. Math. Soc. 14 (1963), 36-43. MR $26 \# 397$.

3. T. G. Hallam, Convergence of solutions of nonlinear differential equations, Ann. Mat. Pura Appl. (to appear).

4. T. G. Hallam and J. W. Heidel, The asymptotic manifolds of a perturbed linear system of differential equations, Trans. Amer. Math. Soc. 149 (1970), 233-241. MR 41 \#2136.

5. T. G. Hallam and V. Lakshmikantham, Growth estimates for convergent solutions of ordinary differential equations, J. Math. Phys. Sci. (to appear).

6. V. Lakshmikantham and S. Leela, Differential and integral inequalities, Theory and Applications, vol. 1, Academic Press, New York, 1969.

7. Ja. D. Mamedov, One-sided estimates in the conditions for existence and uniqueness of solutions of the limit Cauchy problem in a Banach space, Sibirsk. Mat. Ž. 6 (1965), 1190-1196. (Russian) MR $32 \# 7908$. 
8. A. Wintner, Asymptotic equilibria, Amer. J. Math. 68 (1946), 125-132. MR 7,297.

9. - An abelian lemma concerning asymptotic equilibria, Amer. J. Math 68 (1946), 451-454. MR 8,71.

10. - On a theorem of Bôcher in the theory of ordinary linear differential equations, Amer. J. Math. 76 (1954), 183-190. MR 15,426.

DEPARTMENT OF MATHEMATICS, FLORIDA STATE UNIVERSITY, TALLAHASSEE, FLORIDA 32306 